\title{
Molecular Epidemiology of Bloodstream Infection-causing Acinetobacter baumannii from Five Tertiary Hospitals in Beijing, China \\ Ling Guo ${ }^{1}$, Chunmei Bao ${ }^{2}$, Jie Liu ${ }^{3}$, Xiuhong $\mathrm{Hao}^{4}$, Jingui $\mathrm{Cao}^{5}$, Liyan $\mathrm{Ye}^{1}$ and Jiyong Yang ${ }^{*}$
}

${ }^{1}$ Department of Microbiology, Chinese PLA General Hospital, Beijing, 100853, China

${ }^{2}$ Clinical Diagnostic Center, 302nd Hospital of China, 100039, China

${ }^{3}$ Department of Laboratory, PLA Army General Hospital, Beijing, 100700, China

${ }^{4}$ Clinical Laboratory, Navy General Hospital, PLA, Beijing, 100048, China

${ }^{5}$ Department of Infection Control, Air Force General Hospital, PLA, Beijing, 100142, China

Acinetobacter baumannii has emerged as a pathogen that is commonly involved in various nosocomial infections and sometimes community-acquired infections over the last decade. Nosocomial A.baumannii infections mainly include ventilator-pneumonia(VAP), bloodstream infections (BSI), meningitis, and wound infections. Epidemiological studies indicate the mortality rates of $A$. baumannii infections have increased from 10 to $43 \%$ in intensive care units (ICUs) and from 7.8 to $23 \%$ outside ICUs. Multidrug-resistant (MDR) A. baumannii bacteremia usually leads to an even higher mortality $[2,3]$.

Another main reasons to study A. baumannii is its ability to acquire the resistance determinants that caused the emergence of MDR strains and the spread of the pathogen [4]. During the past decade, the terms 'pan-drug resistance', 'extensively drug resistance', and 'MDR' have commonly been applied to A. baumannii to described the phenotypes of resistance to all, to all but one or two, and to three or more classes of potentially effective antimicrobial agents, respectively $[5,6]$. In recent years, the increasing trend of carbapenem resistance in bacteria has become a great concern because it dramatically limits the range of therapeutic alternatives $[7,8]$. The most common mechanism of carbapenem resistance in Acinetobacter spp. is the production of carbapenem-hydrolyzing class

The aims of the present study were: (i) to identify the clonal relationship of A. baumannii strains associated with BSI from Beijing hospitals; (ii) to investigate the susceptibility rates among these strains; and (iii)to identify the carbapenem-hydrolyzing genes carried by these strains. In this study, we collected 97 clinical $A$. baumannii isolates from patients with nosocomial bloodstream infectionfrom five tertiary hospitals in Beijing, China. All A. baumannii isolates were identified by VITEK MALDI-TOF Mass Spectroscopy or VITEKGN identification card (BioMérieux). Antimicrobial susceptibilities were determined by VITEKGN09 cards (BioMérieux) and the results were interpreted according to the CLSI interpretative criteria. Their genetic relationship was analyzed by pulsed-field gel electrophoresis (PFGE) according to the previously described methods [12]. Sequence type (ST) was evaluated by multi loci sequence typing (MLST) following the guidance of Pasteur Institute's MLST method. A multiplex PCR was used to screen the class $\mathrm{D}$ carbapenemase genes including $b l a_{\text {OXA }}$ ${ }_{51}$-like, $b l a_{\text {OXA-23 }}$-like, $b l a_{\text {OXA }-40}-$ like, and $b l a_{\text {OXA-58 }}$-like genes [13]. The presence of other resistance determinants, including $b l a_{\mathrm{SIM}}, b l a_{\mathrm{AIM}}$, $b l a_{\mathrm{IMP}}, b l a_{\mathrm{VIM}}, b l a_{\mathrm{SIM}}$, and $b l a_{\mathrm{NDM}}$, was detected by several multiplex PCR using specific primers as reported previously [14].

PFGE analysis showed that 39 different types have been identified, designated types 1-39. Among them, 52 (53.6\%) isolates belonged to type1 and 45 (46.4\%) belonged to other types. MLST analysis revealed that 73 stains were designated to 9 different existing STs and 24 stains belonged to new STs. The predominant ST, ST699, was found in 65

\section{Publication History:}

Received: November 10, 2017

Accepted: December 28, 2017

Published: December 30, 2017

\section{Keywords:}

Bloodstream infection, Intensive care units, Antimicrobial agents, Drug resistance

isolates. ST63, ST64, ST68, ST338, ST359, ST396, ST667,or ST691 was only found in one isolate. eBURST analysis of the nine existing STs found in this study together with other profiles of the Institute Pasteur's MLST database showed that ST699 belongs to international CC2 [15].

A. baumannii is, in clinical terms, intrinsically resistant to ampicillin, amoxicillin-clavulanate, cefazolin, cefotaxime, ceftriaxone, ertapenem, trimethoprim, and fosfomycin. Antibiotics of choice for the treatment of $A$. baumannii infections include the aminoglycosides, fluoroquinolones, and carbapenems[16]. In our study, of the 97 A. baumannii isolates, $71(73.2 \%)$ were resistant to amikacin, $73(75.26 \%)$ were resistant to ciprofloxacin, and 64 (65.98\%) were resistant to levofloxacin. The whole rate of imipenem susceptibility is $22.68 \%(22 / 97)$, and $17.02 \%$ (8/47), $73.33 \%(11 / 15)$, $27.27 \%(3 / 11), 0 \%(0 / 17)$, and $0 \%(0 / 7)$ among the strains isolated from the five hospitals, respectively. In the first hospital, the major ST type of the resistant isolates is ST699 (37/40), all of which can be detected with the $b l a_{\text {OXA-23 }}$-like gene. The rate of imipenem susceptibility is $73.33 \%(11 / 15)$ in the second hospital, which is significantly higher than those of other hospitals. In addition, there were no ST699 in the isolates from this hospital and most isolates cannot be designated to specific STs except ST667 $(n=1)$ and ST359 (ST=1). This is a hospital for infectious diseases, which might explain why the characteristics of the A. baumannii is different from others in terms of STs and drug resistance. In the other three hospitals, the result was similar to that in the first hospital: the main ST was ST699 and almost all the resistant can be detected with $b l a_{\text {OXA-23 }}$-like gene.

"Corresponding Author: Dr. Jiyong Yang, Department of Microbiology, Chinese PLA General Hospital, Beijing, 100853, China, Tel: +86 10 66936575, E-mail: yangjy301@hotmail.com

Citation: Guo L, Bao C, Liu J, Hao S, Cao J, et al. (2017) Molecular Epidemiology of Bloodstream Infection-causing Acinetobacter baumannii from Five Tertiary Hospitals in Beijing, China. Int J Clin Med Microbiol 2: 127. doi: https://doi. org/10.15344/2456-4028/2017/127

Copyright: (C) 2017 Guo et al. This is an open-access article distributed under the terms of the Creative Commons Attribution License, which permits unrestricted use, distribution, and reproduction in any medium, provided the original author and source are credited. 
Citation: Guo L, Bao C, Liu J, Hao S, Cao J, et al. (2017) Molecular Epidemiology of Bloodstream Infection-causing Acinetobacter baumannii from Five Tertiary Hospitals in Beijing, China. Int J Clin Med Microbiol 2: 127. doi: https://doi.org/10.15344/2456-4028/2017/127

Page 2 of 2

Among the 75 isolates that resist to imipenem, resistance to carbapenems was related to $b l a_{\text {OXA-23 }}-$ like $(\mathrm{n}=66)$, both $b l a_{\text {OXA-23 }}$-like and $b l a_{\mathrm{OXA}-58}-\mathrm{like}(\mathrm{n}=1), b l a_{\mathrm{OXA}-58}-\mathrm{like}(\mathrm{n}=4)$, and $b l a_{\mathrm{NDM}-1}(\mathrm{n}=2)$. No resistance genes that were described previously can be detected in the remaining two isolates.

In conclusion, we have documented the characteristics of BSIcausing A.baumannii isolates from five tertiary hospitals in Beijing, China. The carbapenem-resistant rate ranges from $78.32 \%$ and $91.36 \%$ among the four of five hospitals (no data could be obtained from the second hospital). There were intra-and inter-hospital outbreak spreads and ST699 belonging to international CC2 was the predominant disseminating clones. Further studies are warranted to investigate why the isolates from the second hospital are completely different from the others. The production of CHDLs, especially OXA-23, may play an important role in reducing imipenem susceptibility among the A.baumannii isolates. Our results are concordant with the observations that the $b l a_{\mathrm{OXA}-23}$-like genes have disseminated in medical institutes worldwide including China [17-19]. We additionally identified two $b l a_{\text {NDM-1 }}$-positive A. baumannii isolates. Previous studies showed that the patients with carbapenem-resistant $A$. baumannii bacteremia have higher mortality rate than those with carbapenem-susceptible A. baumannii bacteremia [20]. Therefore, given the high resistance rate to drugs especially carbapenems and the prevalence of highrisk clones, it is necessary to strengthen the monitoring to prevent and control the clinical infection of A.baumannii in nosocomial environment.

\section{Competing Interests}

The authors declares that they have no competing interests.

\section{References}

1. Peleg AY, Seifert $H$, Paterson DL (2008) Acinetobacter baumannii: Emergence of a successful pathogen. Clin Microbiol Rev 21: 538-582.

2. Falagas $M E$, Bliziotis IA, Siempos II (2006) Attributable mortality of acinetobacter baumannii infections in critically ill patients: A systematic review of matched cohort and case-control studies. Crit Care 10: R48.

3. Lee HY, Chen CL, Wu SR, Huang CW, Chiu CH (2014) Risk factors and outcome analysis of acinetobacter baumannii complex bacteremia in critical patients. Crit Care Med 42: 1081-1088.

4. Lockhart SR, Abramson MA, Beekmann SE, Gallagher G, Riedel S, et al. (2007) Antimicrobial resistance among gram-negative bacilli causing infections in intensive care unit patients in the united states between 1993 and 2004. J Clin Microb 45: 3352-3359.

5. Durante-Mangoni E, Zarrilli R (2011) Global spread of drug-resistant acinetobacter baumannii: Molecular epidemiology and management of antimicrobial resistance. Future microbiology 6: 407-422.

6. Magiorakos AP, Srinivasan A, Carey RB, Carmeli Y, Falagas ME, et al. (2012) Multidrug-resistant, extensively drug-resistant and pandrug-resistant bacteria: An international expert proposal for interim standard definitions for acquired resistance. Clin Microbiol Infect 18: 268-281.

7. Poirel L, Nordmann P (2006) Carbapenem resistance in acinetobacter baumannii: Mechanisms and epidemiology. Clin Microbiol Infect 12: 826836.

8. Gupta N, Limbago BM, Patel JB, Kallen AJ (2011) Carbapenem-resistant enterobacteriaceae: Epidemiology and prevention. Clin Infect Dis 53: 60-67.

9. Walther-Rasmussen J, Hoiby N (2006) Oxa-type carbapenemases. J Antimicrob Chemother 57: 373-383.

10. Hu WS, Yao SM, Fung CP, Hsieh YP, Liu CP, Lin JF (2007) An oxa-66/oxa51-like carbapenemase and possibly an efflux pump are associated with resistance to imipenem in acinetobacter baumannii. Antimicrob Agents Chemother 51: 3844-3852.
11. Turton JF, Ward ME, Woodford N, et al. (2006) The role of isaba1 in expression of oxa carbapenemase genes in acinetobacter baumannii. FEMS Microbiol Lett 258: 72-77.

12. Durmaz R, Otlu B, Koksal F, Hosoglu S, Ozturk R, et al. (2009) The optimization of a rapid pulsed-field gel electrophoresis protocol for the typing of acinetobacter baumannii, escherichia coli and klebsiella spp. Jpn $\mathrm{J}$ Infect Dis 62: 372-377.

13. Woodford N, Ellington MJ, Coelho JM, et al. (2006) Multiplex pcr for genes encoding prevalent oxa carbapenemases in acinetobacter spp. Int $J$ Antimicrob Agents 27: 351-353.

14. Poirel L, Walsh TR, Cuvillier V, Nordmann P (2011) Multiplex pcr for detection of acquired carbapenemase genes. Diagn Microbiol Infect Dis 70: 119-123.

15. Diancourt L, Passet V, Nemec A, Dijkshoorn L, Brisse S (2010) The population structure of acinetobacter baumannii: Expanding multiresistant clones from an ancestral susceptible genetic pool. PLoS One 5: e10034.

16. Dijkshoorn L, Nemec A, Seifert H (2007) An increasing threat in hospitals: Multidrug-resistant acinetobacter baumannii. Nat Rev Microbiol 5: 939-951.

17. Fu Y, Zhou J, Zhou H, Yang Q, Wei Z, et al. (2010) Wide dissemination of oxa-23-producing carbapenem-resistant acinetobacter baumannii clonal complex 22 in multiple cities of china. J Antimicrob Chemother 65: 644-650.

18. He C, Xie $Y$, Fan $H$, Kang M, Tao $C$, et al. (2011) Spread of imipenemresistant acinetobacter baumannii of european clone ii in western china. Int J Antimicrob Agents 38: 257-260.

19. Zhong Q, Xu W, Wu Y, Xu H (2012) Clonal spread of carbapenem nonsusceptible acinetobacter baumannii in an intensive care unit in a teaching hospital in china. Ann Lab Med 32: 413-419.

20. Huang ST, Chiang MC, Kuo SC, Lee YT, Chiang TH, et al. (2012) Risk factors and clinical outcomes of patients with carbapenem-resistant acinetobacter baumannii bacteremia. J Microbiol Immunol Infect 45: 356-362. 